\title{
The impact of paratracheal lymph node metastasis in squamous cell carcinoma of the hypopharynx
}

\author{
Young-Hoon Joo $\cdot$ Dong-Il Sun $\cdot$ Kwang-Jae Cho $\cdot$ \\ Jung-Hae Cho $\cdot$ Min-Sik Kim
}

Received: 15 June 2009 / Accepted: 16 November 2009 / Published online: 1 December 2009

(C) The Author(s) 2009. This article is published with open access at Springerlink.com

\begin{abstract}
The aim of this study was to analyze the prevalence and prognostic importance of paratracheal lymph nodes in squamous cell carcinoma of the hypopharynx. A retrospective review of 64 previously untreated patients with squamous cell carcinoma (SCC) of the hypopharynx that underwent surgery was performed. Ipsilateral paratracheal lymph node metastases occurred in 22\% (14 out of 64) and the mean number of paratracheal lymph nodes dissected per side was 2.3 (range 1-6). Contralateral paratracheal lymph node metastases were present in $2 \%$ ( 1 out of 42). Sixty-seven percent with postcricoid SCC and $22 \%$ with pyriform sinus SCC developed clinical node-positive ipsilateral paratracheal lymph node metastases, whereas $11 \%$ with posterior pharyngeal wall SCC developed paratracheal metastases. There was a significant correlation between paratracheal lymph node metastasis and cervical metastasis $(p=0.005)$, and the primary tumor site (postcricoid, 57.1\%; pyriform sinus, 20.0\%; posterior pharyngeal wall, $8.3 \%)(p=0.039)$. Patients with no evidence of paratracheal lymph node metastasis may have a survival benefit (5-year disease-specific survival rate, 60 vs. 29\%). However, this result did not reach statistical significance $(p=0.071)$. The patients with SCC of the postcricoid and/ or pyriform sinus were at risk for ipsilateral paratracheal lymph node metastasis; furthermore, patients with paratracheal node metastasis had a high frequency of cervical metastasis and a poorer prognosis. Therefore, routine ipsilateral paratracheal node dissection is recommended during
\end{abstract}

Y.-H. Joo · D.-I. Sun · K.-J. Cho · J.-H. Cho · M.-S. Kim ( ()

Department of Otolaryngology-Head and Neck Surgery, College of Medicine, Kangnam St. Mary's Hospital,

The Catholic University of Korea, 505 Banpodong,

Seochogu, Seoul 137-040, Korea

e-mail: entkms@catholic.ac.kr; joodoct@yahoo.co.kr the surgical treatment of patients with SCC of the postcricoid and/or pyriform sinus with clinical node metastases.

Keywords Hypopharynx - Squamous cell carcinoma . Lymph nodes $\cdot$ Neck dissection

\section{Introduction}

The hypopharynx is subdivided anatomically into three areas: the pyriform sinus, the posterior pharyngeal wall, and the postcricoid region. Among these sites, carcinomas develop most frequently in the pyriform sinus region. Hypopharyngeal squamous cell carcinoma (SCC) is a very aggressive cancer that is generally diagnosed at advanced stages and consequently has a poor prognosis and low survival. More than $75 \%$ of patients with hypopharyngeal tumors have stage III or IV disease at presentation [1]. Cervical metastases are present in $60-80 \%$ of patients and signify more advanced disease and a poorer prognosis [2, 3]. For this reason, treatment modalities for SCCs at these sites must address the potential for spread of disease to the neck, even for early primary tumors. In this regard, both surgery and radiotherapy are the mainstays of most curative efforts aimed at this disease.

Metastases to the thyroid gland and paratracheal lymph nodes occur in $30 \%$ of patients with hypopharyngeal tumors [4]. A $20 \%$ frequency of occult nodal metastases to ipsilateral paratracheal lymph nodes has been reported in patients with postcricoid lesions and tumors that involve the pyriform fossa apex that are stage N0 [5]. For laryngeal/ hypopharngeal and upper esophageal tumors metastatic disease involving the paratracheal nodal group has been implicated in recurrent disease, particularly in patients with peristomal recurrence; this suggests the prognostic 
significance of positive paratracheal metastatic spread $[6,7]$. Treatment planning for patients with paratracheal metastasis must take into consideration the likelihood of regional recurrence and/or distant metastasis.

The purpose of this study was to analyze the paratracheal nodal yield and frequency of metastases, and determine their relationship with cervical nodal metastases, and overall survival in patients with hypopharyngeal SCC.

\section{Materials and methods}

\section{Patients}

The study group consisted of 64 patients that were not previously treated for SCC of the hypopharynx; they underwent surgery at the Department of Otolaryngology-Head and Neck Surgery, College of Medicine, The Catholic University of Korea, Seoul, Korea, from October 1993 to June 2008. The cases were reviewed retrospectively. The diagnostic evaluations at presentation included a complete physical examination, panendoscopy, CT and/or MRI scans of the head and neck, chest X-rays, and laboratory tests. We assessed the CT and/or MRI to determine the regional lymph node status. The panendoscopy was mainly performed under general anesthesia, and it consisted of a microlaryngoscopy, bronchoscopy, and esophagoscopy. The pharynx and upper esophagus were examined with a rigid hypopharyngoscope. No patient had radiological evidence of distant metastases at presentation, and all patients were treated with curative intent. At our institution, in principal, the choice of radical therapy is surgery, including partial pharyngectomy, and neck lymph node metastasis is treated by neck dissection. Additional radio(chemo)therapy is conducted in patient with multiple lymph nodes metastasis. For histopathologic examination, the formalin-fixed surgical specimen was vertically or horizontally sectioned depending on the extent of tumor involvement. The tissue sections underwent standard histopathologic evaluation and complete histopathologic review was done in all cases by a single pathologist. The median age was 61.0 (range 3475 years); there were 62 males and 2 females. The survival outcomes of surgery group of patients were compared with the outcomes of patients with hypopharyngeal cancer who were treated at our institution with definitive chemoradiotherapy. From October 1993 to June 2008, 38 patients were identified with SCC of the hypopharynx and had been treated with concurrent chemoradiotherapy. Of this group 16 patients were treated with curative intent. The patients were staged according to the 2002 American Joint Committee on Cancer (AJCC) staging system. The follow-up period ranged from 7 to 128 months, with a mean of 36.1 months. The Institutional Review Board of the
Kangnam St Mary's Hospital (Seoul, Korea) approved this retrospective review of the medical records and the use of archived tumor specimens.

\section{Surgical procedures}

For tumor excision, 40 (63\%) patients had conservative surgery with laryngeal preservation, $15(23 \%)$ had total laryngectomy with partial pharyngectomy, $7(11 \%)$ had total laryngopharyngectomy, and $2(3 \%)$ had a total laryngopharyngoesophagectomy. En bloc resection was performed according to the surgical procedures. Laryngeal resection was performed under direct vision based on the invasion of the tumor. The resection of the posterior pharyngeal wall was determined by a safe tumor margin of up to two-thirds of the lateral dimension of the pharyngeal wall. The inferior margin could be $2 \mathrm{~cm}$ below the upper margin of the cervical esophagus without causing any postoperative functional problems. The surgical defects were reconstructed with a radial forearm free flap in 36 cases, pectoralis major myocutaneous flap in 7 cases, anterolateral thigh free flap in 4 cases, and a gastric pull-up in 2 cases. All patients had neck dissection at the time of the primary surgery: a radical neck dissection was performed in 27 cases, a modified radical neck dissection in 20 cases, and a selective (level II-IV) neck dissection in 17 cases. For the 56 contralateral cases, selective (level II-IV) neck dissection was performed in 48 patients and a modified radical neck dissection in 8 . Among the 64 patients that had surgery, all patients had an ipsilateral paratracheal lymph node dissection and 42 patients had bilateral paratracheal node dissections when there was clinical or radiological evidence of disease involvement of the cervical lymph nodes bilaterally. The paratracheal node dissection included dissection of all of the nodes between the carotid artery laterally and the trachea medially, as far inferior into the superior mediastinum as possible, through the cervical approach. There was no perioperative mortality. One patient required re-exploration for vascular compromise of the radial forearm free flap. Two patients developed marginal necrosis of the flap, which resulted in pharyngocutaneous fistulas that were managed by healing with secondary intention after debriding the necrotic tissue in the flap. There were no enteric strictures related to the fistulas. Recurrent laryngeal nerve injury occurred in two patients of the conservative surgery with laryngeal preservation. One patient could be decannulated, whereas the other patients required the retainer.

\section{Postoperative radiotherapy}

Indications and modalities for adjuvant treatments varied over time: positive or close margins found on the resection, advanced $\mathrm{T}$ stage, lymphovascular invasion, perineural 
invasion, multiple nodal metastasis, or extracapsular spread were findings that required additional treatment. Fifty-two (81\%) patients had adjuvant radiotherapy, and 12 (19\%) patients received surgery alone. The radiation techniques used were based on the site of disease and potential areas of spread. Postoperatively, the patients received a total dose of $55-66$ Gy to the site of the lesions with a $1.5-2.0 \mathrm{~cm}$ margin. Treatment was delivered in 2 Gy fractions, with 5 fractions per week.

\section{Statistical analysis}

To determine the significance between paratracheal lymph node metastasis and clinical factors (such as age, gender, primary tumor site, TN stage, tumor cell differentiation, pyriform sinus apex involvement), the chi-square test, Fisher's exact test, multiple logistic regression analysis, multiple linear regression analysis, and correlation analysis were used, as appropriate. The overall survival was determined using the Kaplan-Meier method. The Cox proportional hazard model with likelihood ratio statistics was used to further evaluate each variable by multivariate survival analysis. A $p<0.05$ was considered statistically significant. All calculations were performed using SPSS software ver. 13.0 (SPSS, Chicago, IL, USA).

\section{Results}

Patient characteristics

The site of the original primary tumor was recorded in all patients and included: the pyriform sinus in 45 (70\%), the posterior pharyngeal wall in $12(19 \%)$, and the postcricoid area in $7(11 \%)$. Regarding their pathological stages, there were 2 (3\%), 25 (39\%), 25 (39\%), and $12(19 \%)$ patients with stage T1-T4 cancers, respectively. Concerning the disease stage of the cervical lymph nodes, there were 15 (23\%), $10(16 \%), 37(58 \%)$, and $2(3 \%)$ cases with N0-N3, respectively (Table 1$)$. There were $9(14 \%)$ patients with pyriform sinus apex involvement and $9(14 \%)$ with positive resection margins. Regarding tumor cell differentiation, there were 9 (14\%), $43(67 \%)$, and 12 (19\%) well-differentiated, moderate-differentiated, and poorly differentiated cases, respectively.

\section{Prevalence of paratracheal nodal metastases}

The rate of ipsilateral paratracheal lymph node metastasis was 22\% (14 out of 64) among all cases; 57\% (4 out of 7) had postcricoid carcinoma, 20\% (9 out of 45) had pyriform sinus carcinoma, and $8 \%$ (1 of 11) had posterior pharyngeal wall carcinoma. Contralateral paratracheal lymph node
Table 1 Pathology staging for patients undergoing paratracheal node dissection $(N=64)$

\begin{tabular}{lccccccc}
\hline Stage & N0 & N1 & N2a & N2b & N2c & N3 & Total \\
\hline T1 & 1 & 1 & - & - & - & - & 2 \\
T2 & 5 & 5 & 1 & 10 & 3 & 1 & 25 \\
T3 & 6 & 2 & 2 & 8 & 6 & 1 & 25 \\
T4 & 3 & 2 & 1 & 2 & 4 & - & 12 \\
Total & 15 & 10 & 4 & 20 & 13 & 2 & 64 \\
\hline
\end{tabular}

Table 2 Incidence of paratracheal nodal metastasis by site of primary tumor in patients with hypopharyngeal cancer

\begin{tabular}{lllll}
\hline Level & PS & PPW & PC & Total \\
\hline Ipsilateral $(n=64)$ & & & & \\
$\mathrm{cN}+(n=47)$ & $7 / 32$ & $1 / 9$ & $4 / 6$ & $12 / 47$ \\
$\mathrm{cN} 0(n=17)$ & $2 / 13$ & $0 / 3$ & $0 / 1$ & $2 / 17$ \\
Contralateral $(n=42)$ & & & & \\
$\mathrm{cN}+(n=37)$ & $1 / 28$ & $0 / 6$ & $0 / 3$ & $1 / 37$ \\
$\mathrm{cN} 0(n=5)$ & $0 / 4$ & $0 / 1$ & $0 / 0$ & $0 / 5$ \\
\hline
\end{tabular}

$c N+$ Clinically positive lymph node, $c N O$ clinically negative lymph node

metastases were present in 1 out of the 42 cases (2\%). The mean number of paratracheal lymph nodes dissected per side was 2.3 (range 1-6). The CT and/or MRI detected metastatic cervical disease accurately in 42 out of the 56 sides of the neck $(75 \%)$, whereas they detected metastatic paratracheal lymph node accurately in 8 of the 15 cases $(53 \%)$. Histological paratracheal metastases in the clinically nodepositive neck were more frequent in patients with postcricoid $(67 \%)$ or pyriform sinus hypopharyngeal SCC $(22 \%)$ than in patients with posterior pharyngeal wall SCC (11\%) (Table 2). Patients with SCC of the pyriform sinus had metastatic disease more often; histological metastases were confirmed in $15 \%$ of cases with contralateral dissections, in the clinically node-positive neck, and in $4 \%$ of ipsilateral dissections, in the clinically node-negative neck.

Relationship between paratracheal lymph node metastasis and clinical factors (Table 3.)

We analyzed the correlation between paratracheal lymph node metastasis and several clinical factors among the 64 patients with hypopharyngeal SCC. There was a significant correlation of paratracheal lymph node metastasis with the primary tumor site and $\mathrm{N}$ stage $(p=0.039$ and $p=0.005$, respectively). However, there was no significant relationship between age $(p=0.253)$, gender $(p=0.447)$, T stage $(p=0.862)$, tumor cell differentiation $(p=0.460)$, pyriform sinus apex involvement $(p=0.370)$ and paratracheal lymph node metastasis. The multivariate analysis showed that the 
Table 3 Clinical factors affecting paratracheal lymph node metastasis in patients with hypopharyngeal squamous cell carcinoma $(N=64)$

\begin{tabular}{|c|c|c|c|}
\hline Parameters & $\begin{array}{l}\text { PTN positive } \\
(N=14)\end{array}$ & $\begin{array}{l}\text { PTN negative } \\
(N=50)\end{array}$ & $p$ value \\
\hline Age & & & 0.362 \\
\hline$<60$ & $6(16.7 \%)$ & $30(83.3 \%)$ & \\
\hline$\geq 60$ & $8(28.6 \%)$ & $20(71.4 \%)$ & \\
\hline Gender & & & 1.000 \\
\hline Male & $14(22.6 \%)$ & $48(77.4 \%)$ & \\
\hline Female & $0(0 \%)$ & $2(100 \%)$ & \\
\hline Primary site & & & $0.039 *$ \\
\hline PS & $9(20.0 \%)$ & $36(80.0 \%)$ & \\
\hline PPW & $1(8.3 \%)$ & $11(91.7 \%)$ & \\
\hline $\mathrm{PC}$ & $4(57.1 \%)$ & $3(42.9 \%)$ & \\
\hline T stage & & & 0.862 \\
\hline $\mathrm{T} 1$ & $0(0 \%)$ & $2(100 \%)$ & \\
\hline $\mathrm{T} 2$ & $6(24.0 \%)$ & $19(76.0 \%)$ & \\
\hline $\mathrm{T} 3$ & $5(20.0 \%)$ & $20(80.0 \%)$ & \\
\hline $\mathrm{T} 4$ & $3(25.0 \%)$ & $9(75.0 \%)$ & \\
\hline $\mathrm{N}$ stage & & & $0.005 *$ \\
\hline No & $0(0 \%)$ & $15(100 \%)$ & \\
\hline N1 & $1(10.0 \%)$ & $9(90.0 \%)$ & \\
\hline N2 & $12(32.4 \%)$ & $25(67.6 \%)$ & \\
\hline N3 & $1(50.0 \%)$ & $1(50.0 \%)$ & \\
\hline Histologic grade & & & 0.460 \\
\hline Well differentiated & $1(11.1 \%)$ & $8(88.9 \%)$ & \\
\hline $\begin{array}{l}\text { Moderately } \\
\text { differentiated }\end{array}$ & $9(20.9 \%)$ & $34(79.1 \%)$ & \\
\hline Poorly differentiated & $4(33.3 \%)$ & $8(66.7 \%)$ & \\
\hline Apex involvement & & & 0.397 \\
\hline Yes & $3(33.3 \%)$ & $6(66.7 \%)$ & \\
\hline No & $11(20.0 \%)$ & $44(80.0 \%)$ & \\
\hline
\end{tabular}

$P T N$ paratracheal lymph node, $P S$ pyriform sinus, $P P W$ posterior pharyngeal wall, $P C$ postcricoid

$\mathrm{N}$ stage was significantly correlated with paratracheal lymph node metastasis $(p=0.009)$; however, there was no relationship between paratracheal lymph node metastasis and age, gender, primary tumor site, $\mathrm{T}$ stage, tumor cell differentiation, and pyriform sinus apex involvement.

Relationship between paratracheal lymph node metastasis and survival rate

In the 59 patients followed for more than 12 months, there was a 3-year overall and disease-specific survival of 53 and $56 \%$. The 5-year overall and disease-specific survival rates were 49 and $52 \%$, respectively. In the concurrent chemoradiotherapy group, the 5-year overall survival and diseasespecific survival rate were 46 and $49 \%$, respectively. However, there was no disease-specific survival difference between surgery group and chemoradiotherapy group $(p=0.601)$. The 5-year disease-specific survival among 14 patients with paratracheal lymph node metastasis was $29 \%$, whereas for the 45 metastasis-free patients it was $60 \%$ (Fig. 1). However, this result did not reach statistical significance $(p=0.071)$. Cervical nodal metastasis $(p=0.042)$ and pyriform sinus apex involvement $(p=0.035)$ were statistically significant prognostic factors associated with disease-specific survival. Gender, age, primary tumor site, $\mathrm{T}$ stage, tumor cell differentiation, resected margin involvement, and postoperative radiotherapy were not associated with disease-specific survival. The multivariate survival analysis showed that there was no adverse factor affecting disease-specific survival.

\section{Discussion}

Hypopharyngeal carcinoma has a high tendency to invade cervical lymph nodes. This nodal extension is a wellknown prognostic factor $[8,9]$. The hypopharynx has an extensive lymphatic network. The lymphatic drainage of the hypopharynx is divided into anterior and posterior groups [10]. The anterior collecting system exits, along with the lymphatics of the supraglottic larynx, through the thyrohyoid membrane. These vessels course through prelaryngeal lymph nodes and primarily drain into Groups II and III. The posterior group drains the inferior hypopharynx. These vessels penetrate the superior constrictor muscle and drain into the paratracheal lymph nodes, lateral retropharyngeal lymph nodes and the internal jugular chain [7].

Welsh et al. [11] initially confirmed the importance of the paratracheal nodes in lymphatic drainage of the larynx and hypopharynx. Harrison et al. reported that carcinomas of the subglottic region, trachea, and cervical esophagus are at a high risk for paratracheal and tracheobronchial lymph node metastasis, and recommended resection of the manubrium to remove these lymph nodes and reduce the risk for parastomal disease recurrence [5]. Weber et al. [7] reported on paratracheal lymph node metastasis in patients with tumors of the larynx (metastasis in 17.6\%), pharynx (metastasis in 8.3\%), and esophagus (metastasis in 71.4\%). Some investigators have suggested that ipsilateral paratracheal node dissection should be included as part of a selective neck dissection in all patients with tumors invading the subglottis, pyriform fossa apex, and postcricoid region [12]. In our study, the patients with postcricoid SCC had a significantly higher rate of paratracheal lymph node metastasis compared to patients with pyriform sinus SCC. In addition, patients with posterior pharyngeal wall SCC had the lowest rate of paratracheal lymph node metastasis. Furthermore, paratracheal lymph node metastasis had a significantly 
Fig. 1 Kaplan-Meier diseasespecific survival curve (a) and disease-specific survival curve according to the paratracheal node status (b)

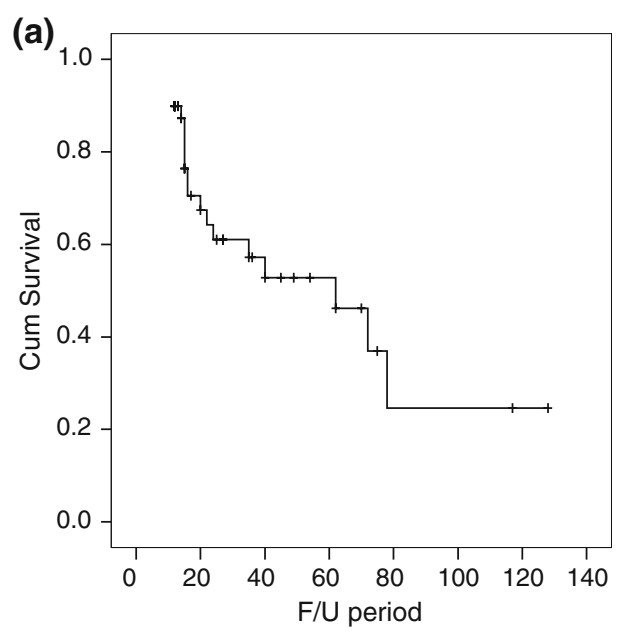

greater risk of cervical lymph node metastases $(p=0.005)$. However, we found no significant correlation between paratracheal lymph node metastasis and age, gender, $\mathrm{T}$ stage, tumor cell differentiation, or pyriform sinus apex involvement.

Advanced-stage neck disease is significantly associated with increased locoregional recurrence rates and poorer 5 -year disease-specific survival rates. N2 or N3 neck disease is associated with 5-year disease-specific survival rates of $0-20 \%$ compared to the $28-57 \%$ in patients with N0 or N1 disease [2, 13]. In a prospective study of 50 patients with carcinoma of the larynx, hypopharynx, and cervical esophagus, Timon et al. [14] reported that the rate of paratracheal nodal metastases was $26 \%$. Moreover, they reported that the survival in patients with positive paratracheal nodes demonstrated a trend towards poorer survival compared to patients without paratracheal nodal involvement, and concomitant involvement of both cervical and paratracheal nodal groups was associated with the poorest survival probability. Previous reports on surgery plus postoperative radiation therapy have shown local recurrence rates of $4-18 \%$, regional recurrence rates of $17-47 \%$ and 5-year disease-specific survival rates of $40-59 \%$ [2, 13, 15]. Koo et al. [16] reported that the rate of distant metastasis $(26 \%)$ was significantly higher than the rate of locoregional recurrence $(9 \%)$ in patients with pyriform sinus carcinoma, and recommended that hypopharyngeal carcinoma should be considered a systemic disease.

The results of this study showed that the patients with paratracheal lymph node metastasis had poorer survival than metastasis-free patients with regard to the 5-year disease-specific survival rates. The rate of the 5-year diseasespecific survival decreased dramatically with the presence of cervical lymph node metastasis and pyriform sinus apex involvement ( $p=0.042$ and 0.035 , respectively) on the univariate analysis, but did not reach significance on the multivariate analysis.

There were some limitations in this study. The main limitation was the retrospective analysis. Second limitation was the small number of patients with paratracheal node metastasis of hypopharyngeal carcinoma. Third, patients with hypopharyngeal carcinoma at different subsites were not equally distributed. Lastly, the median follow-up time of this study was rather short. It was thought that there were limitations of the overall and disease-specific survival data. Further studies with a large number of subjects should be made to confirm our finding that ipsilateral paratracheal lymph node metastasis influences prognosis and to verify the conflicting results between our findings and those of previous studies.

\section{Conclusion}

The findings of this study of 64 patients with previously untreated hypopharyngeal carcinoma that had primary surgery and paratracheal lymph node dissection followed by radiation therapy at a single institution showed a high rate of ipsilateral paratracheal lymph node metastasis in patients with SCC of the postcricoid and/or pyriform sinus with clinically node-positive neck findings; these patients had a poor prognosis. Therefore, these findings suggest the importance of routine ipsilateral paratracheal node dissection with surgical treatment of patients with SCC of the postcricoid and/or pyriform sinus and clinically positive neck nodes.

\section{Conflict of interest statement None.}

Open Access This article is distributed under the terms of the Creative Commons Attribution Noncommercial License which permits any noncommercial use, distribution, and reproduction in any medium, provided the original author(s) and source are credited. 


\section{References}

1. Hoffman HT, Karnell LH, Funk GF, Robinson RA, Menck HR (1998) The National Cancer Data Base Report on cancer of the head and neck. Arch Otolaryngol Head Neck Surg 124:951-962

2. Ho CM, Lam KH, Wei WI, Yuen PW, Lam LK (1993) Squamous cell carcinoma of the hypopharynx-analysis of treatment results. Head Neck 15:405-412

3. Lefebvre JL, Castelain B, DeLaTorre JC, Delobelle-Deroide A, Vankemmel B (1987) Lymph node invasion in hypopharynx and lateral epilarynx: a prognostic factor. Head Neck Surg 10:14-18

4. Wei WI (2002) The dilemma of treating hypopharyngeal carcinoma: more or less. Arch Otolaryngol Head Neck Surg 128:229 232

5. Buckley JG, MacLennan K (2000) Cervical node metastases in laryngeal and hypopharyngeal cancer: a prospective analysis of prevalence and distribution. Head Neck 22:380-385

6. Harris HH, Butler E (1968) Surgical limits in cancer of the subglottic larynx. Arch Otolaryngol 87:64-67

7. Weber RS, Marvel J, Smith P, Hankins P, Wolf P, Goepfert H (1993) Paratracheal lymph node dissection for carcinoma of the larynx, hypopharynx, and cervical esophagus. Otolaryngol Head Neck Surg 108:11-17

8. Driscoll WG, Nagorsky MJ, Cantrell RW, Johns ME (1983) Carcinoma of the pyriform sinus: analysis of 102 cases. Laryngoscope 93:556-560
9. Bataini JP, Bernier J, Brugere J, Jaulerry C, Picco C, Brunin F (1985) Natural history of neck disease in patients with squamous cell carcinoma of oropharynx and pharyngolarynx. Radiother Oncol 3:245-255

10. Lindberg R (1972) Distribution of cervical lymph node metastases from squamous cell carcinoma of the upper respiratory and digestive tracts. Cancer 29:1446-1449

11. Welsh LW (1964) The normal human laryngeal lymphatics. Ann Otol Rhinol Laryngol 73:569-582

12. Harrison DFN (1975) Laryngectomy for subglottic lesions. Laryngoscope 85:1208-1210

13. Kraus DH, Zelefsky MJ, Brock HA, Huo J, Harrison LB, Shah JP (1997) Combined surgery and radiation therapy for squamous cell carcinoma of the hypopharynx. Otolaryngol Head Neck Surg 116:637-641

14. Timon CV, Toner M, Conlon BJ (2003) Paratracheal lymph node involvement in advanced cancer of the larynx, hypopharynx, and cervical esophagus. Laryngoscope 113:1595-1599

15. Ho CM, Ng WF, Lam KH, Wei WI, Yuen AP (1997) Submucosal tumor extension in hypopharyngeal cancer. Arch Otolaryngol Head Neck Surg 123:959-965

16. Koo BS, Lim YC, Lee JS, Kim YH, Kim SH, Choi EC (2006) Management of contralateral N0 neck in pyriform sinus carcinoma. Laryngoscope 116:1268-1272 